\title{
$K$-THEORY AND PATCHING FOR CATEGORIES OF COMPLEXES
}

\author{
STEVEN E. LANDSBURG
}

Consider a pullback square of commutative noetherian rings and surjective homomorphisms

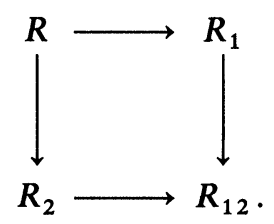

Our goal is to understand certain categories of complexes over $R$ in terms of categories of complexes over the other three rings, and in particular to study the algebraic $K$-theory of these categories.

The classical and best understood example is the category of finitely generated projective $R$-modules, $\mathbf{P}(R)$. From Milnor [M], we know that

(1) the square

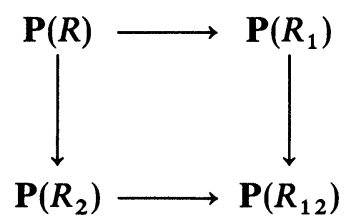

is cartesian; i.e., the map from $\mathbf{P}(R)$ to the fiber product $\mathbf{P}\left(R_{1}\right) \times_{\mathbf{P}\left(R_{12}\right)} \mathbf{P}\left(R_{2}\right)=$ $\left\{\left(P_{1}, P_{2}, \alpha\right) \mid P_{i} \in \mathscr{P}\left(R_{i}\right), \alpha: P_{1} \otimes_{R} R_{2} \rightarrow R_{1} \otimes_{R} P_{2}\right.$ an isomorphism $\}$ is an equivalence of categories; the inverse equivalence is given by pullback, or "patching".

(2) there is a boundary map $\partial: K_{1}\left(\mathbf{P}\left(R_{12}\right)\right) \rightarrow K_{0}(\mathbf{P}(R))$ that is part of an exact Mayer-Vietoris sequence

$$
K_{1}(R) \rightarrow K_{1}\left(R_{1}\right) \oplus K_{1}\left(R_{2}\right) \rightarrow K_{1}\left(R_{12}\right) \stackrel{\partial}{\rightarrow} K_{0}(R) \rightarrow K_{0}\left(R_{1}\right) \oplus K_{0}\left(R_{2}\right) \rightarrow K_{0}(R)
$$

where $K_{i}(S)$ is defined to be $K_{i}(\mathbf{P}(S))$ for any ring $S$. The boundary map $\partial$ is constructed via patching in accordance with condition (1).

For applications in the theory of algebraic cycles (see, e.g., [RCAKT], [AC], [RCG]), one wants similar results for categories of modules having both finite

Received 19 October 1989. Revision received 28 June 1990. 
projective dimension and a given lower bound on codimension, or more generally for categories of modules having both finite projective dimension and support in a specified family of closed subsets. Here the situation is more complicated. Indeed the recent work of Man [Ma, 5.4] shows that a pullback module can have finite projective dimension only under very restrictive conditions on certain Tor groups. This and the results of [PM] suggest that the natural setting for patching is not the category of modules but a corresponding derived category whose objects are complexes of modules.

Thus we consider bounded complexes $P_{i}$ of projective $R_{i}$-modules having all of their homology in a specified category. Given data $\left(P_{1}, P_{2}, \alpha\right)$ where $\alpha: P_{1} \otimes R_{2} \rightarrow$ $R_{1} \otimes_{R} P_{2}$ is a quasi isomorphism, we would like to be able to patch along $\alpha$ to construct a perfect complex over $R$. (Of course this is easy when $\alpha$ is an isomorphism of complexes, but we need the more general formulation.) Following some preliminaries in Section 1, we will show in Section 2 that such a patching exists and is functorial in the patching data.

Next we turn to the construction of a boundary map $K_{n}\left(\mathscr{A}_{12}\right) \rightarrow K_{n-1}(\mathscr{A})$ where $\mathscr{A}_{12}$ and $\mathscr{A}$ are categories of complexes over $R_{12}$ and $R$. Thus we seek to generalize Milnor's result both by considering the $K$-theory of far more general categories and by constructing the boundary map for arbitrary $n$. In Section 3, we define the groups $K_{n}(\mathscr{A})$ as the homotopy groups of a certain simplicial set $T(\mathscr{A})$. In Section 4 , we construct a map

$$
\Omega\left|T\left(\mathscr{A}_{12}\right)\right| \rightarrow|\hat{T}(\mathscr{A})|
$$

where $\hat{T}(\mathscr{A})$ is an approximation to $T(\mathscr{A})$; this induces the desired boundary map. We conclude the section by proving the exactness of a Mayer-Vietoris sequence for lower $K$-theory.

The construction of the map $(*)$ is analogous to Milnor's construction of the boundary map $\partial$ for lower $K$-theory of projective modules. However, there are a number of technical obstacles to be overcome. First, Milnor's construction uses the fact that any free module over $R_{12}$ is the image of a free module over $R_{1}$; no corresponding fact is true for complexes with homology of a specified codimension. However, for any nonliftable complex, we are able to construct a flat family of complexes connecting the given complex to one that is at least liftable up to quasi isomorphism (we call such a flat family a "homotopy"); at this point we use the fact that our patching results are established for quasi isomorphisms and not just for isomorphisms of complexes. Second, the analogue of Milnor's construction yields a map only on one-simplices. Although the maps on higher simplices are described similarly, we need to make a number of technical adjustments in order to get well-defined maps that commute with all of the faces.

A number of the results in this paper appeared previously in my unpublished paper [KTDC]. The patching results in particular are essentially unchanged, except for some minor corrections. However, the $K$-theory in [KTDC] was defined "a la Bass" and in particular only $K_{0}$ and $K_{1}$ were defined. The $K$-theory here is defined 
a la Waldhausen via Hinich and Schechtman. This allows us to use powerful modern machinery (in particular the construction of the loop space by Gillet and Grayson in [GG]) yielding far more general results via somewhat simpler techniques.

I am grateful to the referee for his careful reading.

1. Preliminaries. In this section we give some simple definitions and establish our notation. We also establish our two key technical lemmas in 1.4 and 1.6.

1.1. Definitions. Let $\mathbf{A}$ be an abelian category. An object $R$ of $\mathbf{A}$ is a generator if for each $A \in \mathrm{Ob}(\mathbf{A})$ there exists a cardinal number $K$ and an epimorphism $R^{K} \rightarrow A$. ( $R^{K}$ denotes the direct sum, not the direct product, of $K$ copies of $R$.) $A$ is finitely generated (with respect to $R$ ) if $K$ can be taken to be finite. A projective generator is a generator which is a projective object in A. A projectively generated abelian category is a pair $(\mathbf{A}, R)$ where $\mathbf{A}$ is an abelian category and $R$ is a projective generator in $\mathbf{A}$. We will often abbreviate $(\mathbf{A}, R)$ by just $\mathbf{A}$ when no confusion can result.

1.2. Definitions. Let $\mathbf{A}$ be an additive category. A complex in $\mathbf{A}$ is a diagram

$$
A_{.}=\left(\cdots \longrightarrow A_{n+1} \stackrel{d_{n+1}^{A}}{\longrightarrow} A_{n} \stackrel{d_{n}^{A}}{\longrightarrow} A_{n-1} \longrightarrow \cdots\right)
$$

such that $d_{n}^{A} d_{n+1}^{A}=0$ for all $n$. That is, all complexes are indexed homologically, so that the boundary map has degree - 1 . A complex is bounded below by $N$ if $A_{i}=0$ for all $i<N$, bounded above by $N$ if $A_{i}=0$ for all $i>N$, bounded below if it is bounded below by $N$ for some $N$, and bounded above if it is bounded above by $N$ for some $N$.

A complex $A$, is perfect if there is a bounded complex of finitely generated projective objects $P$. and a quasi isomorphism $P$. $\rightarrow A$.

1.3. Definitions. Let $\mathbf{A}$ be an abelian category and let $\mathbf{A}^{\prime}$ be any subcategory. We define the following categories of complexes.

$\mathscr{C}(\mathbf{A})=$ the category of all complexes in $\mathbf{A}$.

$\mathscr{C}\left(\mathbf{A}, \mathbf{A}^{\prime}\right)=$ the category of all those complexes in $\mathbf{A}$ that have all of their homology objects in $\mathbf{A}^{\prime}$.

$\mathscr{C}_{+}(\mathbf{A})=$ the category of all those complexes in $\mathbf{A}$ that are bounded below.

$\mathscr{C}_{+}\left(\mathbf{A}, \mathbf{A}^{\prime}\right)=\mathscr{C}_{+}(\mathbf{A}) \cap \mathscr{C}\left(\mathbf{A}, \mathbf{A}^{\prime}\right)$.

$\mathscr{C}_{b}(\mathbf{A})=$ the category of all those complexes in $\mathbf{A}$ that are bounded both below and above.

$\mathscr{C}_{b}\left(\mathbf{A}, \mathbf{A}^{\prime}\right)=\mathscr{C}_{b}(\mathbf{A}) \cap \mathscr{C}\left(\mathbf{A}, \mathbf{A}^{\prime}\right)$

Let $\mathbf{P} \subset \mathbf{A}$ be any subcategory. Then we write $\mathscr{C}(\mathbf{P})$ for the category of complexes over $P$. We write

$$
\begin{aligned}
& \mathscr{C}\left(\mathbf{P}, \mathbf{A}^{\prime}\right)=\mathscr{C}(\mathbf{P}) \cap \mathscr{C}\left(\mathbf{A}, \mathbf{A}^{\prime}\right), \\
& \mathscr{C}_{+}(\mathbf{P})=\mathscr{C}(\mathbf{P}) \cap \mathscr{C}_{+}(\mathbf{A}),
\end{aligned}
$$

and similarly for $\mathscr{C}_{+}\left(\mathbf{P}, \mathbf{A}^{\prime}\right), \mathscr{C}_{b}(\mathbf{P})$, and $\mathscr{C}_{b}\left(\mathbf{P}, \mathbf{A}^{\prime}\right)$. 
Now suppose in addition that $\mathbf{A}$ is projectively generated and $\mathbf{P}$ is the category of projective objects in $\mathbf{A}$. Then we write

$\mathscr{C}_{b}(\mathbf{P})_{N}^{*}=$ the full subcategory of $\mathscr{C}_{b}(\mathbf{P})$ consisting of those complexes $P$. such that $P_{i}=0$ whenever $i>N$ and $P_{i}$ is free whenever $i<N$. (We make no new assumption about $P_{N}$.)

$\mathscr{C}_{b}\left(\mathbf{P}, \mathbf{A}^{\prime}\right)_{N}^{*}=\mathscr{C}_{b}\left(\mathbf{P}, \mathbf{A}^{\prime}\right) \cap \mathscr{C}_{b}(\mathbf{P})_{N}^{*}$

We also write

$\mathscr{C}(\mathbf{A})_{\text {per }}=$ the category of all perfect complexes in $\mathscr{C}(\mathbf{A})$,

and for any subcategory $\mathscr{B} \subset \mathscr{C}(\mathbf{A})$ we write

$\mathscr{B}_{\text {per }}=\mathscr{C}(\mathscr{B}) \cap \mathscr{C}(\mathbf{A})_{\text {per }}$.

1.4. Lemma. Let $\mathbf{A}$ be an abelian category and $\mathbf{P}$ the subcategory of projective objects. Let $f: P . \rightarrow Q$. be a quasi isomorphism in $\mathscr{C}_{+}(\mathscr{P})$.

Then there exist acyclic complexes $P_{.}^{\prime}, Q_{.}^{\prime} \in \mathscr{C}_{+}(\mathbf{P})$, and an isomorphism of complexes $\tilde{f}: P . \oplus P^{\prime} \rightarrow Q . \oplus Q^{\prime}$ such that the diagram

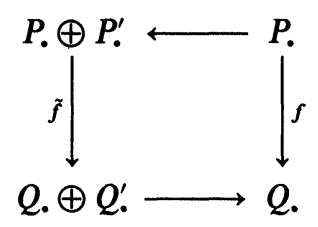

commutes.

Moreover, if $P$. and $Q$. are in $\mathscr{C}_{b}(\mathbf{P})$, then we can take $P_{.}^{\prime}$ and $Q^{\prime}$. to be in $\mathscr{C}_{b}(\mathbf{P})$ as well.

Proof. Let $g: Q . \rightarrow P$. be a homotopy inverse for $f$ and choose maps $\tau: P_{i} \rightarrow P_{i+1}$, $\sigma: Q_{i} \rightarrow Q_{i+1}$ such that

$$
\begin{gathered}
d^{P} \tau+\tau d^{P}=1-g f \\
d^{Q} \sigma+\sigma d^{Q}=1-f g
\end{gathered}
$$

Let $X_{n}=P_{n} \oplus Q_{n-1} \oplus P_{n-2} \oplus \cdots$ and $Y_{n}=Q_{n} \oplus P_{n-1} \oplus Q_{n-2} \oplus \cdots$ (Note that both sums are finite.)

Define $\alpha_{n}: X_{n} \rightarrow Y_{n}$ and $\beta_{n}: Y_{n} \rightarrow X_{n}$ by the matrices

$$
\alpha_{n}=\left(\begin{array}{ccccc}
f & \sigma & 0 & 0 & \ldots . \\
d^{P} & -g & \tau & 0 & \ldots . \\
0 & d^{Q} & f & \sigma & \ldots . \\
0 & 0 & d^{P} & -g & \ldots . \\
\vdots & \vdots & \vdots & \vdots & \\
0 & 0 & 0 & 0 & \ldots .
\end{array}\right) \quad \beta_{n}=\left(\begin{array}{ccccc}
g & \tau & 0 & 0 & \ldots \\
d^{Q} & -f & \sigma & 0 & \ldots \\
0 & d^{P} & g & \tau & \ldots \\
0 & 0 & d^{Q} & -f & \ldots \\
\vdots & \vdots & \vdots & \vdots & \\
0 & 0 & 0 & 0 & \ldots .
\end{array}\right)
$$




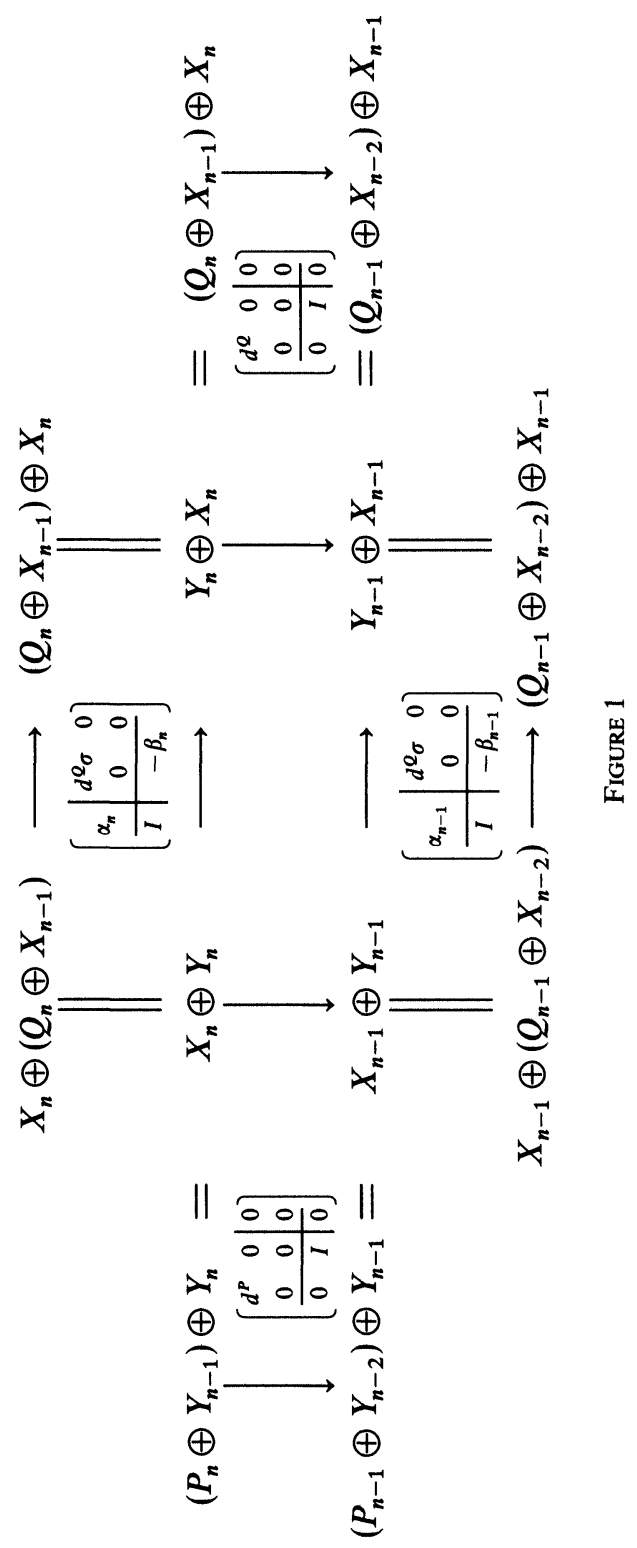


Notice that

$$
\alpha_{n} \beta_{n}=\left(\begin{array}{ccccc}
1-d^{Q} \sigma & & & & \\
& 1 & & * & \\
& & 1 & & \\
0 & & & \ddots & \\
& & & & 1
\end{array}\right) \text { and } \beta_{n} \alpha_{n}=\left(\begin{array}{ccccc}
1-d^{P} \tau & & & \\
& 1 & & * & \\
& & 1 & & \\
0 & & \ddots & \\
& & & & 1
\end{array}\right) \text {. }
$$

Now consider the diagram in Figure 1. It is not hard to check that this diagram commutes; in fact the composition in either direction is

$$
\left[\begin{array}{ccc|c}
d^{Q} & d^{Q} \sigma & 0 & 0 \\
0 & 0 & 0 & \\
\hline d^{P} & -\beta_{n-1} & 0
\end{array}\right]
$$

Therefore, we get a map of complexes

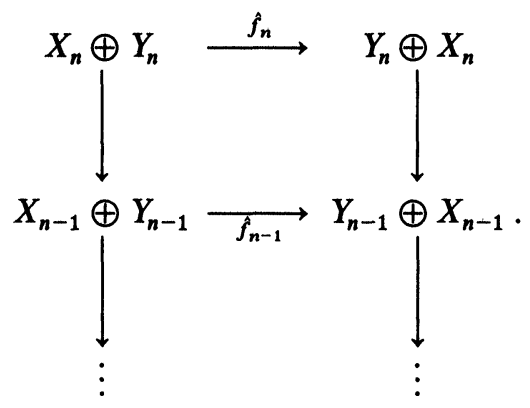

The complex on the left is of the form $P . \oplus P^{\prime}$ and the complex on the right is of the form $Q . \oplus Q^{\prime}$. where

$$
P_{.}^{\prime}=\left(\cdots \underset{\left(\begin{array}{ll}
0 & 0 \\
I & 0
\end{array}\right)}{\longrightarrow} Y_{n-1} \oplus Y_{n} \underset{\left(\begin{array}{ll}
0 & 0 \\
I & 0
\end{array}\right)}{\longrightarrow} Y_{n-2} \oplus Y_{n-1} \underset{\left(\begin{array}{ll}
0 & 0 \\
I & 0
\end{array}\right)}{\longrightarrow} \cdots\right)
$$

and

$$
Q_{.}^{\prime}=\left(\cdots \underset{\left(\begin{array}{ll}
0 & 0 \\
1 & 0
\end{array}\right)}{\longrightarrow} X_{n-1} \oplus X_{n} \underset{\left(\begin{array}{ll}
0 & 0 \\
1 & 0
\end{array}\right)}{\longrightarrow} X_{n-2} \oplus X_{n-1} \underset{\left(\begin{array}{cc}
0 & 0 \\
1 & 0
\end{array}\right)}{\longrightarrow} \cdots\right) .
$$

The acyclicity of these complexes is obvious. 
We must check that $\tilde{f}_{n}$ is an isomorphism. This follows from the observation that

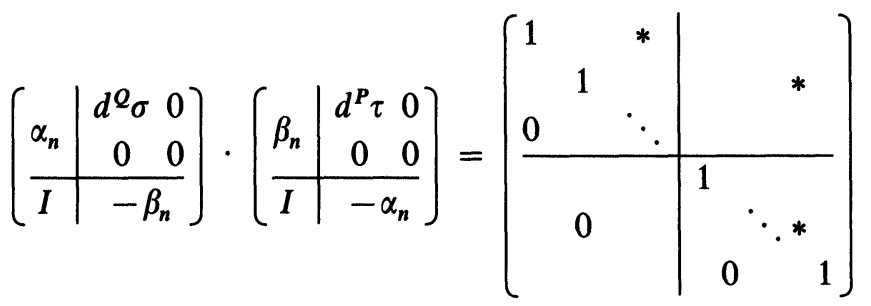

(using our earlier observation about the forms of $\alpha_{n} \beta_{n}$ and $\beta_{n} \alpha_{n}$ ).

Now suppose that $P$. and $Q$. are bounded above by $N$. Then the diagram



is commutative. Thus we can redefine

$$
P_{N}^{\prime}=Y_{N-1} \quad Q_{N}^{\prime}=X_{N-1} \quad P_{N+k}^{\prime}=Q_{N+k}^{\prime}=0 \quad \text { for } k>0,
$$

thereby replacing $P^{\prime}$ and $Q^{\prime}$. by bounded complexes. (Note that $\alpha_{N}=\beta_{N}=1$ since $\sigma_{N}=\tau_{N}=0$.)

1.5. Corollary. Suppose in Lemma 1.4 that $\mathbf{A}$ is projectively generated. Let $\mathbf{B} \stackrel{\varphi}{\rightarrow} \mathbf{A}$ be any map of projectively generated abelian categories preserving the distinguished generator, and let $\mathbf{Q} \subset \mathbf{B}$ be the full subcategory of projective objects in $\mathbf{B}$. Suppose that for some $N>0, P$. and $Q$. are in $\mathscr{C}_{b}(\mathbf{P})_{N}^{*}$ (as defined in 1.3). Then $P^{\prime}$ and $Q^{\prime}$. can be taken to lift to $\mathscr{C}_{b}(\mathbf{Q})$.

Indeed, this is clear since we have

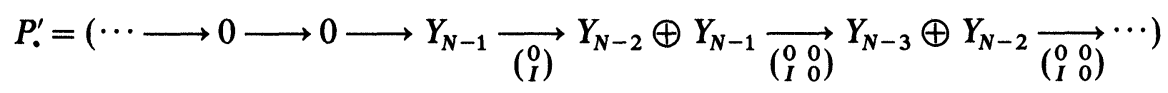

with each $Y_{k}$ a direct sum of the free modules $P_{i}$ and $Q_{i}, i \leqslant k<N$. A similar remark applies to $Q^{\prime}$. The corollary follows immediately.

1.6. Triangles and homotopies. In this section, we restrict attention to the case where $\mathbf{A}$ is given as the category of modules over some ring $R$. We write $\mathbf{A}[t]$ for the category of modules over $R[t]$. If $M$ and $N$ are complexes in $\mathscr{C}(\mathbf{A})$, then a homotopy from $M$ to $N$ is a complex $H$ in $\mathscr{C}(\mathbf{A}[t])$ such that neither $t$ nor $1-t$ is a zero-divisor on $H$, together with specified quasi isomorphisms $M \rightarrow H / t H$ and 
$N \rightarrow H /(1-t) H$. We will denote such a homotopy by the diagram

$$
H \int_{N}^{M}
$$

Homotopies form a category in an obvious way. If $H$ is a homotopy from $M$ to $N$ and $H^{\prime}$ is a homotopy from $M^{\prime}$ to $N^{\prime}$, then a map of homotopies consists of maps $H \rightarrow H^{\prime}, M \rightarrow M^{\prime}$ and $N \rightarrow N^{\prime}$ satisfying the natural compatibility conditions.

A multistep homotopy is a sequence of complexes $H_{1}, H_{2}, \ldots, H_{n}$ in $\mathbf{A}[t]$ such that neither $t$ nor $1-t$ is a zero-divisor on any $H_{i}$, together with specified quasi isomorphisms $M \rightarrow H_{1} / t H_{1}$ and $N \rightarrow H_{n} /(1-t) H_{n}$, and for each $i(1 \leqslant i \leqslant n-1)$ a specified quasi isomorphism in either direction connecting $H_{i} /(1-t) H_{i}$ to $H_{i+1} / t H_{i+1}$.

A structured triangle in $\mathscr{C}_{+}(\mathbf{A})$ is a sequence of maps

$$
\text { A. } \stackrel{f}{\rightarrow} B_{\bullet} \stackrel{g}{\rightarrow} C_{\bullet} \rightarrow \text { Cone }(g) .
$$

in which the last map is the canonical one, together with a specified quasi isomorphism of complexes Cone $(f) . \rightarrow C$. such that the obvious diagram

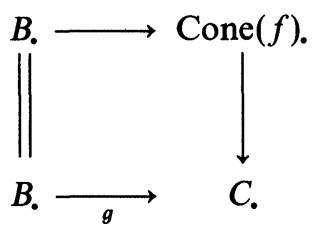

commutes.

We will represent structured triangles by sequences like (1.6.1), or even just

$$
A_{.} \stackrel{f}{\rightarrow} B_{.} \stackrel{g}{\rightarrow} C_{\bullet},
$$

always remembering that part of the structure is suppressed in the notation.

In conjunction with the theory of derived categories, one calls a sequence of three maps a triangle if it is quasi-isomorphic to a standard mapping cone sequence

$$
A_{.} \rightarrow B_{.} \rightarrow C_{\bullet} \rightarrow A[1] \text {. }
$$

There is a forgetful functor from structured triangles to triangles. This functor is neither one-to-one nor onto.

Given a structured triangle (1.6.1), one constructs functorially a map

$$
A[1] . \rightarrow \operatorname{Cone}(g) \text {. }
$$


as follows: the given quasi isomorphism Cone $(f), \rightarrow C$, is given in degree $n$ by a map $\left(g_{n}, h_{n}\right): B_{n} \oplus A_{n-1} \rightarrow C_{n}$. Define (1.6.2) in degree $n$ by the map

$$
\left(\begin{array}{c}
h_{n} \\
f_{n-1}
\end{array}\right): A_{n-1} \rightarrow C_{n} \oplus B_{n-1} \text {. }
$$

For any subcategory $\mathscr{B} \subset \mathscr{C}(A)$, we define $K_{0}(\mathscr{B})$ to be the group with the objects of $\mathscr{B}$ as generators and with a relation $\left[A_{0}\right]+\left[C_{0}\right]=\left[B_{0}\right]$ for each structured triangle (1.6.1) in $\mathscr{B}$.

Traditionally, one defines $K_{0}(\mathscr{B})$ via the same relations, but allowing (1.6.1) to be any triangle. To see that the two definitions coincide, it suffices to show that quasi-isomorphic objects $A$. and $B$. yield the same class in our definition of $K_{0}$. For this it suffices to consider the case where there exists a quasi isomorphism $A_{.} \rightarrow B_{\text {. }}$. Since the cone of such a quasi isomorphism is acyclic, it suffices to show that acyclic objects $X$. give the zero class in $K_{0}$. But this follows because

$$
0 \rightarrow 0 \rightarrow X
$$

can be made into a structured triangle in an obvious (and unique) way.

LEMMA. There is a functor from the category of structured triangles in $\mathscr{C}_{+}(A)$ to the category of homotopies of objects in $\mathscr{C}_{+}(A)$, which maps each triangle (1.6.1) to a homotopy from $A .[1] \oplus B$. to $C_{.}$. The same is true with $\mathscr{C}_{+}(-)$replaced by $\mathscr{C}_{b}(-)$ or by $\mathscr{C}_{+}(-)_{\text {per }}$.

Proof. Map (1.6.1) to $H$., where $H$. is determined by the condition that the diagram



is a homotopy pushout.

Note that $H_{\text {. }} / t H_{\text {. }}$ is canonically isomorphic to $B_{\text {. }} \oplus \operatorname{Cone}(g)$. and $B_{.} \oplus A .[1]$ maps to this by (1.6.3). Also we have a map $C_{\text {. }} \rightarrow H_{\text {. }} /(1-t) H_{\text {. given by reducing the right }}$ vertical map $\bmod (1-t)$. Thus the structured triangle yields a homotopy. This construction extends to a functor on the category of triangles by the universal property of the homotopy pushout.

Corollary. Suppose that $\left[M_{.}\right]=\left[N_{.}\right]$in $K_{0}\left(\mathscr{C}_{+}(\mathbf{A})\right)$. Then there exists a (possibly multistep) homotopy from $M_{0}$ to $N_{0}$ in $\mathscr{C}_{+}(\mathbf{A}[t])$, and similarly, with $\mathscr{C}_{+}(-)$ replaced by $\mathscr{C}_{b}(-)$ or $\mathscr{C}_{b}(-)_{\text {per }}$. 
The functor constructed in the lemma behaves well in a number of ways that we will not list formally but will use as the need arises. For example, if there is an upper bound on the codimension of support of the homology of the complexes appearing in a triangle, then that same upper bound applies to the codimension of support of the homology of the corresponding homotopy.

2. Patching. In this section, we will consider a commutative square of abelian categories which allows "Milnor patching" of projective objects along isomorphisms. We shall show that such a square also allows the patching of complexes of finite projective dimension along quasi isomorphisms.

2.1. Definitions. Let $\mathbf{P}, \mathbf{P}_{1}, \mathbf{P}_{2}$, and $\mathbf{P}_{12}$ be categories and let

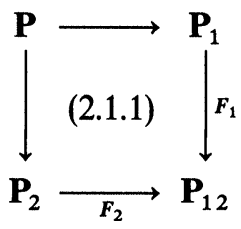

be a commutative square of functors. We define the pseudofiber product category $\mathbf{P}_{1} \times{ }_{\mathbf{P}_{12}} \mathbf{P}_{2}$ as follows. An object is a triple $\left(P_{1}, P_{2}, \alpha\right)$ where $P_{i} \in \mathbf{P}_{i}$ and $\alpha: F_{1} P_{1} \rightarrow F_{2} P_{2}$ is an isomorphism in $\mathbf{P}_{12}$. A morphism $\left(P_{1}, P_{2}, \alpha\right) \rightarrow\left(P_{1}^{\prime}, P_{2}^{\prime}, \alpha^{\prime}\right)$ is a pair of morphisms $P_{i} \rightarrow P_{i}^{\prime}$ making the obvious diagram commute.

There is an obvious functor $\mathbf{P} \rightarrow \mathbf{P}_{1} \times_{\mathbf{P}_{12}} \mathbf{P}_{2}$. We say that the diagram (2.1.1) is pseudocartesian if this functor is an equivalence of categories.

2.2. Definitions. Let $\mathbf{A}, \mathbf{A}_{1}, \mathbf{A}_{2}$ and $\mathbf{A}_{12}$ be abelian categories with projective generators $R, R_{1}, R_{2}$ and $R_{12}$ respectively. Suppose that we are given two commutative squares of functors


and natural transformations $\xi_{i}: 1_{\mathrm{A}_{i}} \Rightarrow H_{i} F_{i}$.

We assume that the functors in (2.2.1) are right exact and take the given projective generators to each other. Let $\mathbf{P} \subset \mathbf{A}, \mathbf{P}_{1} \subset \mathbf{A}_{1}, \mathbf{P}_{2} \subset \mathbf{A}_{2}$ and $\mathbf{P}_{12} \subset \mathbf{A}_{12}$ be the full subcategories of projective objects. Then (2.2.1) induces a diagram

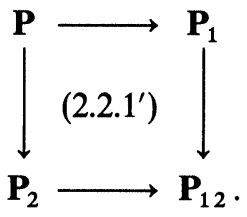


We say that (2.2.1), (2.2.2) and the $\xi_{i}$ constitute a Milnor Patching Situation if the following two conditions hold:

(1) the diagram $\left(2.2 .1^{\prime}\right)$ is pseudocartesian (i.e., the natural map

$$
\theta: \mathbf{P} \rightarrow \mathbf{P}_{1} \times_{\mathbf{P}_{12}} \mathbf{P}_{2}
$$

is an equivalence of categories) and

(2) the inverse equivalence to $\theta$ is given by

$$
\left(P_{1}, P_{2}, \alpha\right) \stackrel{\psi}{\rightarrow} \operatorname{ker}\left(\left(J_{1} P_{1} \oplus J_{2} P_{2}\right) \underset{\left(J_{1} H_{1} \alpha \circ J_{1} \xi_{1}\left(P_{1}\right),-J_{2} \xi_{2}\left(P_{2}\right)\right)}{\longrightarrow} J_{2} H_{2} F_{2} P_{2}\right) .
$$

Note that the kernel in condition (2) is not a priori in $\mathbf{P}$, so that the definition of a Milnor Patching Situation requires both that $\psi$ take its values in $\mathbf{P}$ and that it be inverse to $\theta$.

2.3. Suppose that (2.2.1), (2.2.2) and the $\xi_{i}$ constitute a Milnor Patching Situation. Consider the category of "patching data"

$$
\mathscr{G}=\left\{\left(P_{1 .}, P_{2 .}, \alpha_{\bullet}\right) \mid P_{i \bullet} \in \mathscr{C}_{b}\left(\mathbf{P}_{i}\right), \alpha: F_{1} P_{1 \bullet} \rightarrow F_{2} P_{2} \text { a quasi isomorphism }\right\}
$$

(Recall that $\mathscr{C}_{b}\left(\mathbf{P}_{i}\right)$ consists of bounded complexes of objects in $\mathbf{P}_{i}$.)

Thinking of an object as a complex centered in degree zero gives natural inclusions $\mathbf{P}_{1} \times{ }_{\mathbf{P}_{12}} \mathbf{P}_{2} \rightarrow \mathscr{G}$ and $\mathbf{P} \rightarrow \mathscr{C}_{b}(\mathbf{A})_{\text {per }}$. We now show that the Milnor patching process for projective modules extends to complexes.

THEOREM. Suppose that $\xi_{2}\left(R_{2}\right): R_{2} \rightarrow H_{2}\left(R_{12}\right)$ is an epimorphism. Then there is a functor $\varphi: \mathscr{G} \rightarrow \mathscr{C}_{b}(\mathbf{A})_{\text {per }}$ making the diagram



commute.

Proof. $\operatorname{Map} \mathscr{G} \stackrel{\varphi}{\rightarrow} \mathscr{C}_{b}(\mathbf{A})$ by

$$
\left(P_{1 .}, P_{2 .}, \alpha_{\bullet}\right) \stackrel{\varphi}{\rightarrow} \operatorname{ker}\left(\left(J_{1} P_{1} \bullet \oplus J_{2} P_{2} .\right) \underset{\left(J_{1} H_{1} \alpha \circ J_{1} \xi_{1}\left(P_{1} \cdot\right),-J_{2} \xi_{2}\left(P_{2} \cdot\right)\right)}{\longrightarrow} J_{2} H_{2} F_{2} P_{2}\right)
$$

We must show that the image of $\varphi$ is actually contained in $\mathscr{C}_{b}(\mathbf{A})_{\text {per }}$.

For any sufficiently large $N$, it is easy to construct acyclic complexes $Q_{i}$ in $\mathbf{P}_{i}$. such that $P_{i \bullet} \oplus Q_{i \bullet}$ is in $\mathscr{C}_{b}\left(\mathbf{P}_{i}\right)_{N}^{*}$ (as defined in 1.3) for both $i=1$ and $i=2$. Applying Lemma 1.5 to the map 


$$
F_{1}\left(P_{1} \oplus Q_{1}\right) \underset{\left(\begin{array}{cc}
\alpha & 0 \\
0 & 0
\end{array}\right)}{\longrightarrow} F_{2}\left(P_{2} \oplus Q_{2}\right),
$$

we conclude that there is a commutative diagram of complexes of projective objects

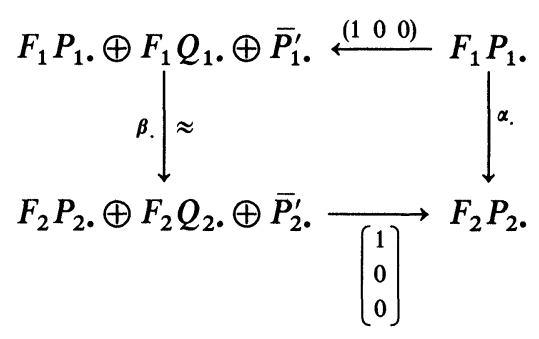

where the $\bar{P}_{i .}^{\prime}$ are bounded acyclic complexes of finitely generated free objects. By Corollary 1.5 , we may assume that $\bar{P}_{i}^{\prime}$ lifts to a complex $P_{i}^{\prime} \in \mathscr{C}_{b}\left(\mathbf{P}_{i}\right)$.

Write $P_{i}^{\prime \prime}=Q_{i} \oplus P_{i}^{\prime}$. Then we have a diagram in which the rows are exact and the vertical arrows are quasi isomorphisms:

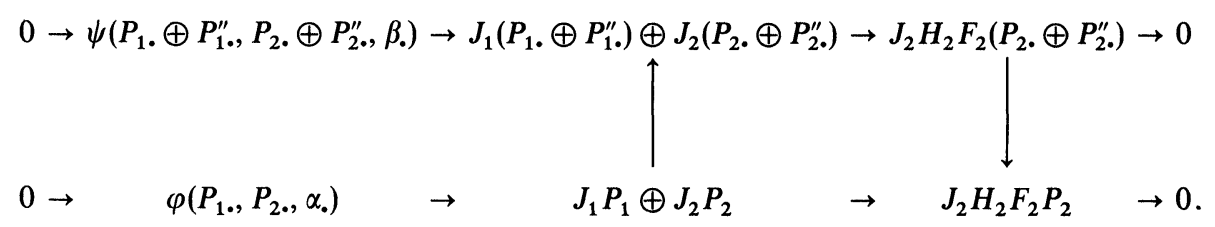

(The surjectivity on the right follows from the assumption that $\xi_{2}\left(R_{2}\right)$ is surjective together with the right exactness of all the functors.)

It follows that $\varphi\left(P_{1 .}, P_{2 .}, \alpha_{\bullet}\right)$ is quasi-isomorphic to $\psi\left(P_{1} \oplus P_{1 .}^{\prime \prime}, P_{2 .} \oplus P_{2 .}^{\prime \prime}, \beta_{\bullet}\right)$. In particular, $\varphi$ takes its values in $\mathscr{C}_{b}(\mathbf{A})_{\text {per }}$.

Corollary. Let $\mathbf{A}^{\prime} \subset \mathbf{A}, \mathbf{A}_{1}^{\prime} \subset \mathbf{A}_{1}$, etc., be abelian subcategories closed under extensions and mapped to each other by the functors. Let $\mathscr{G}^{\prime}=\left\{\left(P_{1 .}, P_{2 .}, \alpha_{\bullet}\right) \in \mathscr{G} \mid P_{i \bullet} \in\right.$ $\left.\mathscr{C}_{b}\left(\mathbf{P}_{i}, \mathbf{A}_{i}^{\prime}\right)\right\}$. Then $\varphi$ restricts to a well-defined functor $\varphi^{\prime}: \mathscr{G}^{\prime} \rightarrow \mathscr{C}_{b}\left(\mathbf{A}, \mathbf{A}^{\prime}\right)_{\text {per }}$.

2.4. We remark that $\varphi$ is naturally quasi-isomorphic to the functor $\tilde{\varphi}: \mathscr{G} \rightarrow$ $\mathscr{C}_{b}(\mathbf{A})_{\text {per }}$ given by taking $\left(P_{1 .}, P_{2 .}, \alpha_{\bullet}\right)$ to

$$
\text { Cone }\left(\left(J_{1} P_{1} . \oplus J_{2} P_{2} \cdot\right) \frac{}{\left(J_{1} H_{1} \propto \circ J_{1} \xi_{1}\left(P_{1}\right),-J_{2} \xi_{2}\left(P_{2} \cdot\right)\right)} J_{2} H_{2} F_{2} P_{2} .\right)[-1]
$$

It follows from the theorem that this complex is perfect. Under the conditions of the corollary, there is a corresponding mapping $\tilde{\varphi}^{\prime}: \mathscr{G}^{\prime} \rightarrow \mathscr{C}_{b}\left(\mathbf{A}, \mathbf{A}^{\prime}\right)_{\text {per }}$. In much of the sequel, we will find it convenient to work with $\tilde{\varphi}$ instead of $\varphi$.

3. Higher $K$-theory. Let $X$ be a noetherian scheme. Our goal in this section is to define the higher $K$-theory of $X$ with respect to a system of supports. We will 
write $M(X)$ for the category of coherent modules on $X$ and $\mathbf{P}(X) \subset \mathbf{M}(X)$ for the subcategory of locally free coherent sheaves.

3.1. The Hinich-Schechtman T-construction. Let $n \geqslant 0$ be an integer. The set $T_{n}(X)$ is defined as follows. An element of $T_{n}(X)$ consists of:

(1) for each pair $(i, j)$ with $0 \leqslant i \leqslant j \leqslant n$, a complex $H_{i j}$ in $\mathscr{C}_{b}(\mathbf{P}(X))$;

(2) a commutative diagram of complexes



(3) a quasi isomorphism (Cone $\left.\left(H_{i j} \rightarrow H_{i k}\right)\right) \rightarrow H_{j k}$ whenever $0 \leqslant i \leqslant j \leqslant k \leqslant n$, subject to various compatibility conditions that are listed in [HS, 3.3].

In particular, each sequence

$$
H_{i j} \rightarrow H_{i k} \rightarrow H_{j k}
$$

is a structured triangle in the sense of (1.6).

Such a simplex will often be denoted by its top row, suppressing references to the remainder of its structure. In that case we also suppress the subscript 0 and write

$$
H_{0} \rightarrow H_{1} \rightarrow H_{2} \rightarrow H_{3} \rightarrow \cdots \rightarrow H_{n}
$$

The sets $T_{n}$ fit together to form a simplicial set, where $d_{i}(i \geqslant 0)$ is given by deleting all of the complexes whose first or second index is $i$.

In [HS, Theorem 3.4], it is shown that the simplicial set $T_{0}(X)$ (which is $T_{0}^{d}(\mathbf{P}(X)$ ) in the notation of [HS]) computes the $K$-theory of $X$; that is,

$$
K_{i}(X)=\pi_{i+1}(T .(X))
$$

3.2. We will want to work with arbitrary perfect complexes. Therefore, we consider the simplicial set $\tilde{T}_{.}(X)$ defined as follows: $\tilde{T}_{n}(X)$ is defined exactly as 
is $T_{n}(X)$, except that the complexes $P_{i j}$ are allowed to be arbitrary objects in $\mathscr{C}_{+}(\mathbf{M}(X))_{\text {per }}$.

Thus there is an obvious inclusion

$$
T_{.}(X) \rightarrow \tilde{T}_{\cdot}(X)
$$

Proposition. The inclusion $T_{0}(X) \rightarrow \tilde{T}_{0}(X)$ is a homotopy equivalence.

Proof. It is shown in [HS] that $T_{.}(X)$ is homotopy equivalent to the simplicial category $S_{0}\left(\mathscr{C}_{b}(\mathbf{P}(X))\right)$, where $S$. is the construction of Waldhausen ([W]), defining the cofibrations to be the monomorphisms. (This is a special case of [HS, Thm. 3.4.].) The same proof shows that $\tilde{T}(X)$ is homotopy equivalent to the simplicial category $S_{.}\left(\mathscr{C}_{+}(\mathbf{M}(X))_{\text {per }}\right)$. Thomason $([\mathrm{T}, 1.9 .1])$ shows that the hypotheses of Waldhausen's Approximation Theorem $([\mathrm{W}, 1.6 .7])$ are satisfied by the inclusion

$$
S_{.}\left(\mathscr{C}_{b}(\mathbf{P}(X))\right) \rightarrow S_{.}\left(\mathscr{C}_{+}(\mathbf{M}(X))_{\mathrm{per}}\right)
$$

and the Approximation Theorem allows us to conclude that the inclusion is a homotopy equivalence.

COROLlary. $\quad K_{i}(X)=\pi_{i+1}(\tilde{T} .(X))$.

3.3. Definitions. Let $\Phi$ be a family of supports in $X$. Let $\mathbf{M}^{\Phi}(X) \subset \mathbf{M}(X)$ consist of those coherent modules whose support is in $\Phi$. For any subcategory $\mathbf{N} \subset \mathbf{M}(X)$, we write

$$
\begin{aligned}
& \mathscr{C}_{+}^{\Phi}(\mathbf{N})=\mathscr{C}_{+}\left(\mathbf{N}, \mathbf{M}^{\Phi}(X)\right) \\
& \mathscr{C}_{b}^{\Phi}(\mathbf{N})=\mathscr{C}_{b}\left(\mathbf{N}, \mathbf{M}^{\Phi}(X)\right)
\end{aligned}
$$

where the right-hand categories are as defined in 1.3.

We define the $K$-theory of $X$ with supports in $\Phi$ by replacing the category $\mathscr{C}_{b}(\mathbf{P}(X))$ with the category $\mathscr{C}_{b}^{\Phi}(\mathbf{P}(X))$ in the Hinich-Schechtman $T$-construction. This gives a new simplicial set $T_{0}^{\Phi}(X)$, and we define

$$
K_{i}^{\Phi}(X)=\pi_{i+1}\left(T_{\bullet}^{\Phi}(X)\right) .
$$

We may also consider the simplicial set $\tilde{T}_{\cdot}^{\Phi}(X)$ constructed by replacing the category $\mathscr{C}_{+}(\mathbf{M}(X))_{\text {per }}$ with the category $\mathscr{C}_{+}^{\Phi}(\mathbf{M}(X))_{\text {per }}$ in the $\tilde{T}$-construction of 3.2 . The proof of the proposition in 3.2 also shows that the inclusion $T_{0}^{\Phi}(X) \rightarrow \widetilde{T}_{0}^{\Phi}(X)$ is a homotopy equivalence, so that we may write

$$
K_{i}^{\Phi}(X)=\pi_{i+1}\left(\tilde{T}_{\cdot}^{\Phi}(X)\right) .
$$

3.4. Using the techniques of [GG], we can construct a simplicial set $G_{.}^{\Phi}(X)$ whose realization is the loop space of the realization of $T_{0}^{\Phi}(X)$. 
For each $n$, an element of $G_{n}^{\Phi}(X)$ consists of two elements $x, y \in T_{n+1}^{\Phi}(X)$ such that

(a) $d_{0}(x)=d_{0}(y)$ and

(b) $d_{1} d_{2} \ldots d_{n} d_{n+1}(x)=d_{1} d_{2} \ldots d_{n} d_{n+1}(y)=0$.

In condition (b), 0 refers to the zero-simplex represented by the zero complex.

We represent such an element as follows: Suppose that the diagrams representing $x$ and $y$ are

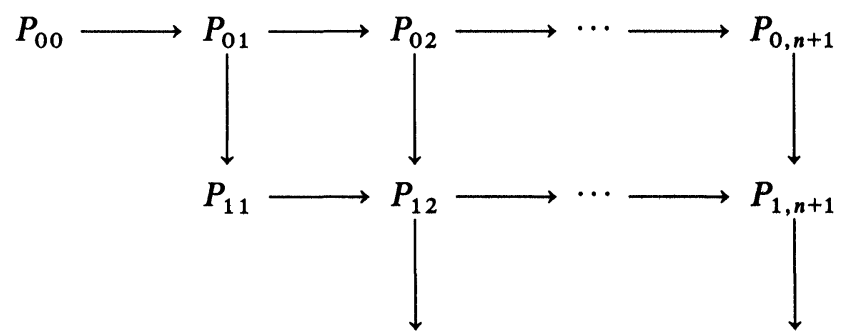

and

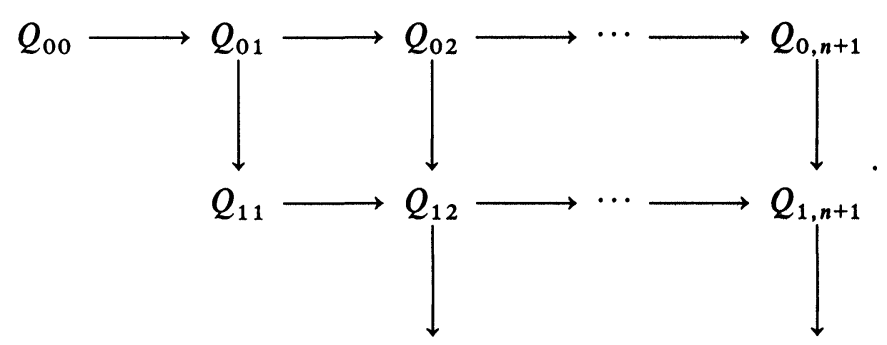

Then for the element $(x, y) \in G_{n}^{\Phi}(X)$, we write

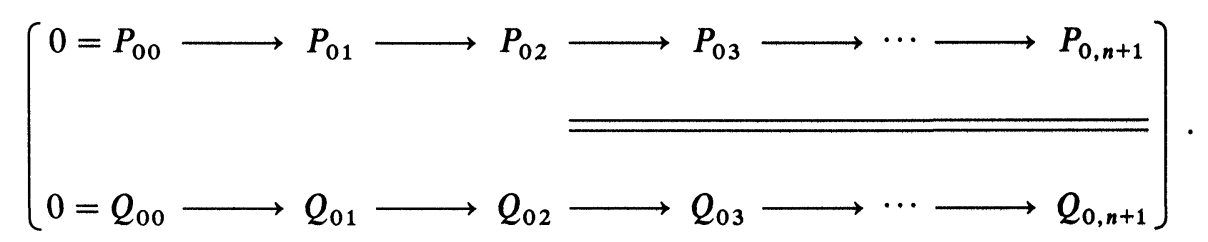

Thus part of the structure of $(x, y)$ is suppressed in the notation. In particular, the structure provides preferred quasi isomorphisms between the cones of $P_{0 i} \rightarrow P_{0 j}$ and of $Q_{0 i} \rightarrow Q_{0 j}$; more precisely, it provides quasi isomorphisms from each of these cones to a given complex $P_{i j}=Q_{i j}$.

It is convenient to reindex so that an $n$-simplex of $G_{.}^{\Phi}(X)$ involves no indices greater than $n$. We do this by setting $P_{i}=P_{0, i+1}$ and $Q_{i}=Q_{0, i+1}$. Thus we will write a typical $n$-simplex as 


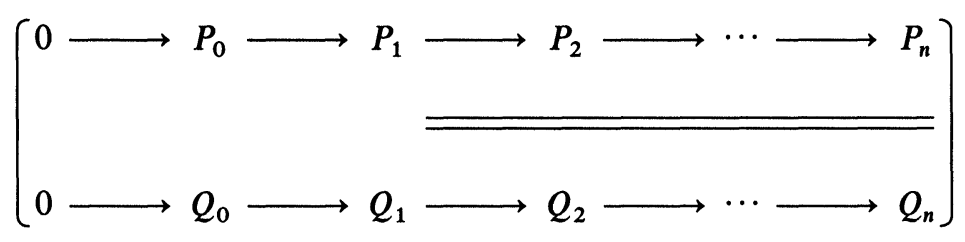

where there are given quasi isomorphisms from the cones of $P_{i} \rightarrow P_{j}$ and $Q_{i} \rightarrow Q_{j}$ to a common object $P_{i j}=Q_{i j}(0 \leqslant i \leqslant j)$. With this notation the face operator $d_{i}$ just eliminates $P_{i}$ and $Q_{i}$.

3.5. THEOREM. There is a homotopy equivalence

$$
\left|G_{\bullet}^{\Phi}(X)\right| \cong \Omega\left|T_{\bullet}^{\Phi}(X)\right|
$$

Consequently,

$$
K_{i}^{\Phi}(X) \cong \pi_{i}\left(G_{\bullet}^{\Phi}(X)\right)
$$

Proof. The idea is to mimic the proof of Theorem 3.1 in [GG]. One replaces exact sequences by triangles, isomorphisms by quasi isomorphisms, quotients by cones, and pushouts by homotopy pushouts throughout.

3.6. We will be interested in the connected component in $G_{.}^{\Phi}(X)$ of the zero simplex

$$
\left[\begin{array}{l}
0 \longrightarrow 0 \\
=0 \\
0 \longrightarrow 0
\end{array}\right]
$$

We denote this connected component by $G_{.}^{\Phi}(X)_{0}$.

The following proposition is easily verified.

Proposition. If the simplex

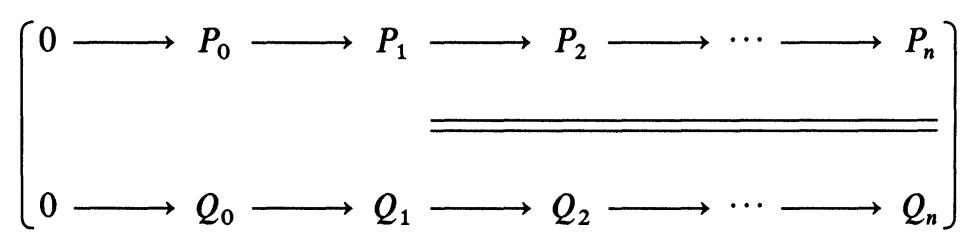

is in $G_{.}^{\Phi}(X)_{0}$, then $\left[P_{i}\right]=\left[Q_{i}\right]$ in $K_{0}\left(\mathscr{C}_{b}^{\Phi}(\mathbf{P}(X))\right)$ for all $i$.

\section{4. $K$-Theory of rings.}

4.1. Throughout this section, we will consider a pullback square of noetherian commutative rings 


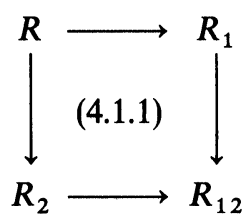

in which all maps are surjective. We assume given families of supports $\Phi, \Phi_{i}(i=1$, 2 , or 12) in $\operatorname{Spec}(R)$ and the $\operatorname{Spec}\left(R_{i}\right)$ satisfying the condition that every element of $\Phi_{1}$ and $\Phi_{2}$ restricts to an element of $\Phi_{12}$, and a closed set $V \subset \operatorname{Spec}(R)$ is in $\Phi$ if and only if it restricts to elements of $\Phi_{1}$ and $\Phi_{2}$.

We will write $\tilde{T}_{.}^{\Phi}(R)$ and $G_{.}^{\Phi}(R)$ for $\widetilde{T}_{.}^{\Phi}(R)(\operatorname{Spec}(R))$ and $G_{.}^{\Phi}(\operatorname{Spec}(R))$. (See 3.3 and 3.4 for definitions.) Likewise for $R_{1}, R_{2}$ and $R_{12}$. For $i=1,2$, or $R_{12}$, we will write $\tilde{T}_{.}^{\Phi}\left(R_{i}\right)$ and $G_{0}^{\Phi}\left(R_{i}\right)$ instead of $\tilde{T}_{\bullet}^{\Phi_{i}}\left(R_{i}\right)$ and $G_{.}^{\Phi_{i}}\left(R_{i}\right)$.

Define $R^{(n)}$ to be the pullback of the diagram

$$
R_{1} \rightarrow R_{12} \leftarrow R_{12}[t] \rightarrow R_{12} \leftarrow R_{12}[t] \rightarrow \cdots \rightarrow R_{12} \leftarrow R_{12}[t] \rightarrow R_{12} \leftarrow R_{2}
$$

where $R_{12}[t]$ occurs $n$ times, each leftward arrow $R_{12} \leftarrow R_{12}[t]$ takes $t$ to 0 , and each rightward arrow $R_{12}[t] \rightarrow R_{12}$ takes $t$ to 1 .

Define a family of supports in $\operatorname{Spec}\left(R^{(n)}\right)$, which will also be denoted $\Phi$, by the condition that $V$ is in $\Phi$ if and only if it restricts to elements of $\Phi_{1}$ and $\Phi_{2}$ in $\operatorname{Spec}\left(R_{1}\right)$ and $\operatorname{Spec}\left(R_{2}\right)$, and to the inverse image of an element of $\Phi_{12}$ in each of the copies of $\operatorname{Spec}\left(R_{12}[t]\right)$.

There is an exact functor $s_{i}^{n}: \mathbf{M}\left(R^{(n)}\right) \rightarrow \mathbf{M}\left(R^{(n+1}\right)(0 \leqslant i \leqslant n)$ given by "insertion of a trivial homotopy" on the $(i+1)^{\text {th }}$ copy of $R_{12}[t]$. This induces a map of simplicial sets $\tilde{T}_{0}^{\Phi}\left(R^{(n)}\right) \rightarrow \tilde{T}_{0}^{\Phi}\left(R^{(n+1)}\right)$. We define

$$
\hat{T}_{\bullet}^{\Phi}(R)=\lim _{\rightarrow} \tilde{T}_{\cdot}^{\Phi}\left(R^{(n)}\right)
$$

and

$$
\hat{K}_{i}^{\Phi}(R)=\pi_{i}\left(\hat{T}^{\Phi}(R)\right)
$$

with the limit being taken over all of the $s_{i}^{n}$.

This notation suppresses the fact that $\hat{T}$ and $\hat{K}$ depend not only on $R$, but on the way $R$ is expressed as a pullback.

4.2. We will define a simplicial map

$$
G_{\cdot}^{\Phi}\left(R_{12}\right)_{0} \rightarrow \hat{T}_{\bullet}^{\Phi}(R)
$$

The full definition is given in Section 4.4, and the reader may skip to that section now if he wishes. Here and in 4.3, we will describe the map on 1-simplices and on 
2-simplices, with the goal of explaining the flavor of the construction before the notation becomes too cumbersome. (The map on 0 -simplices is trivial because $\hat{T}_{.}^{\Phi}(R)$ has only one 0 -simplex.)

For every pair of complexes $(P, Q)$ in $\mathscr{C}_{b}^{\Phi}(\mathbf{P}(X))$ such that $[P]=[Q]$ in $K_{0}\left(\mathscr{C}_{b}^{\Phi}(\mathbf{P}(X))\right)$, use 1.6 to choose arbitrarily and once for all a (possibly multistep) homotopy from $P \oplus Q[1]$ to an acyclic object. Call this homotopy $\alpha(P, Q)$.

Now let

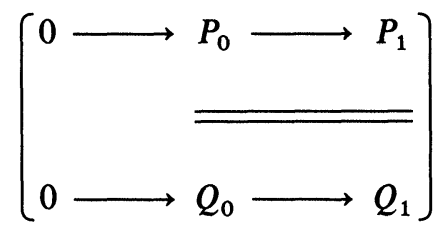

be a 1-simplex in $G_{.}^{\Phi}\left(R_{12}\right)_{0}$. Recall that this notation suppresses a reference to a complex $P_{01}=Q_{01}$, and structured triangles

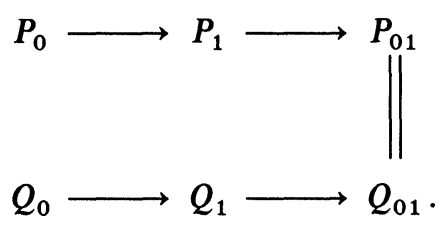

From these structured triangles and (1.6), we construct homotopies

$$
\left\{\begin{array}{l}
P_{0}[1] \oplus P_{1} \\
P_{01}=Q_{01} \\
Q_{0}[1] \oplus Q_{1} .
\end{array}\right.
$$

This gives a complex on "two copies of $R_{12}[t]$ pasted together". To this complex, we add on a direct summand consisting of two copies of the trivial homotopy from $P_{1}[1] \oplus P_{0}$ to itself. That is, we consider

$$
\left\{\begin{array}{l}
P_{0}[1] \oplus P_{1} \oplus P_{1}[1] \oplus P_{0} \\
\left(P_{01}=Q_{01}\right) \oplus P_{1}[1] \oplus P_{0} \\
Q_{0}[1] \oplus Q_{1} \oplus P_{1}[1] \oplus P_{0} .
\end{array}\right.
$$

By 3.6 and 1.6, the top "endpoint" is homotopic to an acyclic complex via $\alpha\left(P_{0}, P_{0}\right) \oplus \alpha\left(P_{1}, P_{1}\right)$, and the bottom endpoint is homotopic to an acyclic com- 
plex via $\alpha\left(P_{0}, Q_{0}\right) \oplus \alpha\left(Q_{1}, P_{1}\right)$. Adjoining these homotopies at the endpoints, we have

$$
\left\{\begin{array}{l}
\text { acyclic } \\
P_{0}[1] \oplus P_{1} \oplus P_{1}[1] \oplus P_{0} \\
\left(P_{01}=Q_{01}\right) \oplus P_{1}[1] \oplus P_{0} \\
Q_{0}[1] \oplus Q_{1} \oplus P_{1}[1] \oplus P_{0} \\
\text { acyclic. }
\end{array}\right.
$$

We can now paste copies of $R_{1}$ and $R_{2}$ at the endpoints as specified in the definition of $R^{(n)}$ and use the functor $\tilde{\varphi}$ of 2.4 to patch the acyclic endpoints of the above diagram to the zero complexes on $R_{1}$ and $R_{2}$.

The resulting object $H$ lies in $\mathscr{C}_{b}\left(\mathbf{M}\left(R^{(n)}\right)\right)_{\text {per }}$ for some sufficiently large $n$ (in fact, we can take $n=4)$, and therefore $0 \rightarrow H$ is a 1 -simplex in $\widehat{T} .(R)$.

4.3. Here we define the map $G_{2}^{\Phi}\left(R_{12}\right)_{0} \rightarrow \widehat{T}_{2}^{\Phi}(R)$.

Continue to choose homotopies as in the second paragraph of 4.2 .

Now let

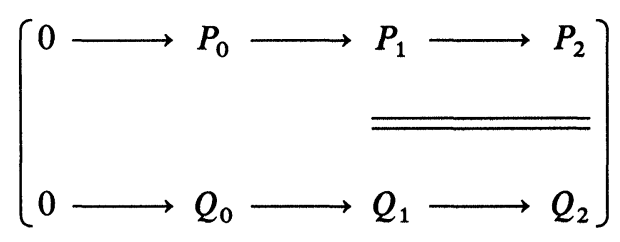

be a 2-simplex in $G_{.}^{\Phi}\left(R_{12}\right)_{0}$.

From the triangles that are part of the simplex (but suppressed in the notation), we use 1.6 to construct homotopies

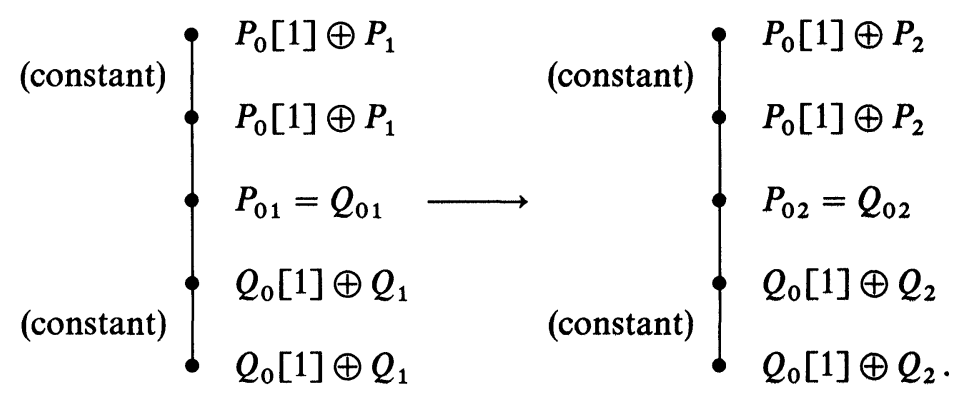


Patching the endpoints of these homotopies together along the canonical identifications gives two complexes on "multiple copies of $R_{12}[t]$ pasted together". We call these complexes $J_{1}$ and $J_{2}$.

The map from $J_{1}$ to $J_{2}$ is constructed as follows. At the topmost "vertex", the map is $\left(\begin{array}{ll}1 & 0 \\ 0 & 0\end{array}\right)$. Along the topmost "edge", it is $\left(\begin{array}{ll}1 & 0 \\ 0 & t f\end{array}\right)$, where we think of " $t=0$ " as the upper vertex and $f: P_{1} \rightarrow P_{2}$ is given as part of the structure of the simplex. Thus at the second vertex from the top, the map is $\left(\begin{array}{ll}1 & 0 \\ 0 & f\end{array}\right)$. Along the second edge, the map is induced by the map of structured triangles

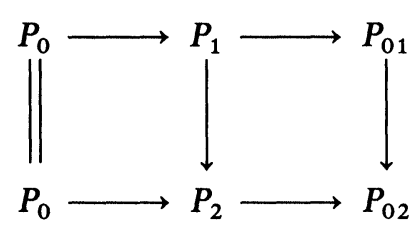

using the functoriality of the construction in (1.6). Thus at the vertex $P_{01}$, the map $P_{01} \rightarrow P_{02}$ is the map given as part of the structure of the simplex. Along the edges and vertices below the center, the map is defined symmetrically.

Set

$$
J_{12}=\left\{\begin{array}{l}
P_{1}[1] \oplus P_{2} \\
P_{12} \\
P_{12} \\
P_{12} \\
Q_{1}[1] \oplus Q_{2}
\end{array}\right.
$$

where all homotopies except the top and bottom ones are constant. Then we have a triangle

$$
J_{1} \rightarrow J_{2} \rightarrow J_{12}
$$

We want to extend these complexes "upward" and "downward" to acyclic complexes so that they can be patched to the zero complexes on $R_{1}$ and $R_{2}$. To accomplish this, add to $J_{1}$ the trivial homotopy from $P_{0} \oplus P_{1}[1]$ to itself, to $J_{2}$ the trivial homotopy from $P_{0} \oplus P_{2}[1]$ to itself, and to $J_{12}$ the trivial homotopy from $P_{1} \oplus P_{2}[1]$ to itself. Then adjoin the following homotopies (which exist by 3.6 and 1.6). 
At the top of $J_{1}, \alpha\left(P_{0}, P_{0}\right) \oplus \alpha\left(P_{1}, P_{1}\right)$.

At the top of $J_{2}, \alpha\left(P_{0}, P_{0}\right) \oplus \alpha\left(P_{2}, P_{2}\right)$.

At the top of $J_{12}, \alpha\left(P_{1}, P_{1}\right) \oplus \alpha\left(P_{2}, P_{2}\right)$.

At the bottom of $J_{1}, \alpha\left(P_{0}, Q_{0}\right) \oplus \alpha\left(Q_{1}, P_{1}\right)$.

At the bottom of $J_{2}, \alpha\left(P_{0}, Q_{0}\right) \oplus \alpha\left(Q_{2}, P_{2}\right)$.

At the bottom of $J_{12}, \alpha\left(Q_{1}, P_{1}\right) \oplus \alpha\left(Q_{2}, P_{2}\right)$.

The maps $J_{1} \rightarrow J_{2} \rightarrow J_{12}$ extend in obvious ways after these adjustments are made. The map $\alpha(M, N) \rightarrow \alpha\left(M^{\prime}, N^{\prime}\right)$ is always zero unless $(M, N)=\left(M^{\prime}, N^{\prime}\right)$, in which case it is the identity.

Finally, adjoin copies of $R_{1}$ and $R_{2}$ at the top and bottom and use the functor $\tilde{\varphi}$ of 2.4 to patch the acyclic endpoints of the $J$ 's to the zero complexes on $R_{1}$ and $R_{2}$. Call the resulting complexes $H_{1}, H_{2}$ and $H_{12}$. Then there is a natural arrow $H_{1} \rightarrow H_{2}$ and a natural map from the cone of this arrow to $H_{12}$. These data fit together to describe a 2-simplex

$$
0 \rightarrow H_{1} \rightarrow H_{2}
$$

in $\hat{T}^{\Phi}(R)$.

4.4. Here we give a complete description of the simplicial map

$$
G_{.}^{\Phi}\left(R_{12}\right) \rightarrow \tilde{T}_{\cdot}^{\Phi}(R)
$$

First, for every pair of complexes $(P, Q)$ in $\mathscr{C}_{b}^{\Phi}(\mathbf{P}(X))$ such that $[P]=[Q]$ in $K_{0}\left(\mathscr{C}_{b}^{\Phi}(\mathbf{P}(X))\right)$, choose arbitrarily and once for all a (possibly multistep) homotopy from $P[1] \oplus Q$ to an acyclic object. Call this homotopy $\alpha(P, Q)$.

Now let

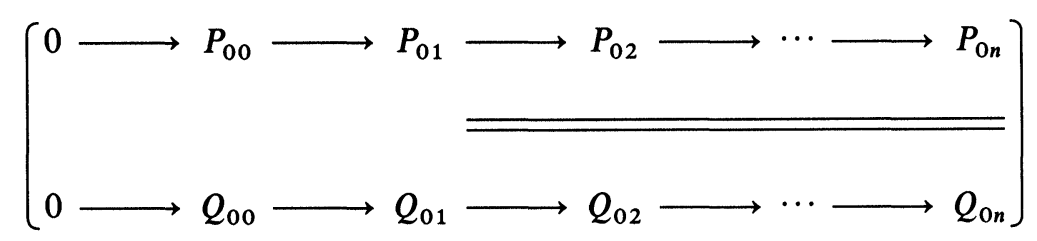

be a simplex in $G_{.}^{\Phi}\left(R_{12}\right)_{0}$. Recall that this notation suppresses references to other complexes $P_{i j}(0<i \leqslant j \leqslant n)$ and maps between them.

For each pair of indices $(i, j)$ with $0 \leqslant i \leqslant j \leqslant n$, consider the sequence of homotopies 


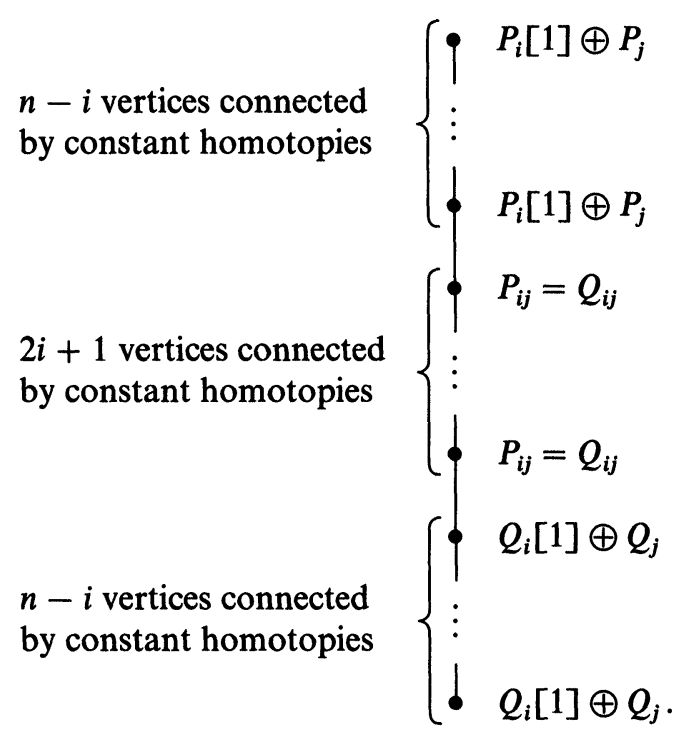

The two nonconstant homotopies are constructed from the triangles that are given as part of the structure of the simplex. Patching these homotopies along the canonical identifications at their endpoints, we construct a complex on "multiple copies of $R_{12}$ pasted together". We call this complex $J_{i j}$.

Next we shall define some maps between the $J_{i j}$. In what follows, we will use the symbol $f$ to denote any map $P_{i j} \rightarrow P_{k l}$ that occurs as part of the structure of the originally given simplex.

For $i \leqslant j \leqslant k$, we define a map $J_{i j} \rightarrow J_{i k}$ as follows.

On the uppermost $n-j$ vertices, the map $P_{i}[1] \oplus P_{j} \rightarrow P_{i}[1] \oplus P_{k}$ is given by the matrix $\left(\begin{array}{ll}1 & 0 \\ 0 & 0\end{array}\right)$. On the constant homotopies connecting these vertices, the map is a constant homotopy from this map to itself.

Moving down to the next $j-i$ vertices, the map $P_{i}[1] \oplus P_{j} \rightarrow P_{i}[1] \oplus P_{k}$ is given by the matrix $\left(\begin{array}{ll}1 & 0 \\ 0 & f\end{array}\right)$. On the homotopies between these vertices, the map is a constant homotopy from this map to itself. On the single homotopy that connects vertex $n-j$ to vertex $n-j+1$ (counting down from the top), the map is given by the matrix $\left(\begin{array}{cc}1 & 0 \\ 0 & t f\end{array}\right)$. (We view the top of the homotopy as the locus of " $t=0$ ".)

On the vertices where the map goes from $P_{i j}$ to $P_{i k}$, it is the map $f$ given by the structure of the simplex, and on the edges between them, it is a constant homotopy from this map to itself.

On the edge that connects vertices $n-i$ and $n-i+1$, the map arises functorially from the map of triangles 




On the remaining vertices, the map is "symmetric"; that is, one makes the same constructions, replacing $P$ 's with $Q$ 's and counting up from the bottom instead of down from the top.

This completes the construction of the map $J_{i j} \rightarrow J_{i k}$.

Next we construct a map $J_{i j} \rightarrow J_{i+1, j}(0 \leqslant i \leqslant i+1 \leqslant j \leqslant n)$.

On the uppermost $n-i-1$ vertices, the map $P_{i}[1] \oplus P_{j} \rightarrow P_{i+1}[1] \oplus P_{j}$ is given by the matrix $\left(\begin{array}{ll}0 & 0 \\ 0 & 1\end{array}\right)$. On the constant homotopies connecting these vertices, the map is a constant homotopy from this map to itself.

On vertex number $n-1$, the map $P_{i}[1] \oplus P_{j} \rightarrow P_{i+1, j}$ is given by the matrix $(0 f)$. On the edge that connects vertex $n-i-1$ to vertex $n-i$, the map arises naturally from the construction of the target homotopy. On the vertices where the map goes from $P_{i j}$ to $P_{i k}$, it is the map $f$ given by the structure of the simplex, and on the edges between them, it is a constant homotopy from this map to itself.

On the edge that connects vertices $n-i$ and $n-i+1$, the map arises canonically from the construction of the homotopy along this edge.

On the remaining vertices, the map is "symmetric"; that is, one makes the same constructions, replacing $P$ 's with $Q$ 's and counting up from the bottom instead of down from the top.

This completes the construction of the map $J_{i j} \rightarrow J_{i+1, j}$.

Maps $J_{i j} \rightarrow J_{k l}(i \leqslant j \leqslant l, i \leqslant k \leqslant l)$ can be constructed as compositions of the maps constructed above. It is easy to see that these maps are well defined (that is, they do not depend on the factorization of $J_{i j} \rightarrow J_{k l}$ ) and that they satisfy the Hinich-Schechtman compatibility conditions.

Next, we alter $J_{i j}$ by adding to it as a direct sum a sequence of constant homotopies from $P_{i} \oplus P_{j}[1]$ to itself. We extend the maps $J_{i j} \rightarrow J_{k l}$ by defining a map on the new summands: it is given by the matrix $\left(\begin{array}{cc}\delta_{i k} & 0 \\ 0 & \delta_{j l}\end{array}\right)$ where $\delta_{m n}$ is the identity if $m=n, 0$
otherwise.

Next, we alter $J_{i j}$ again by adjoining $\alpha\left(P_{i}, P_{i}\right) \oplus \alpha\left(P_{j}, P_{j}\right)$ at the top, and adding $\alpha\left(Q_{i}, P_{i}\right) \oplus \alpha\left(Q_{j}, P_{j}\right)$ at the bottom. The endpoints of these agree canonically with the previously existing endpoints and so can be patched on. We extend the maps $J_{i j} \rightarrow J_{k l}$ to these new "attachments" via the matrix $\left(\begin{array}{cc}\delta_{i k} & 0 \\ 0 & \delta_{j l}\end{array}\right)$.

This makes the endpoints of the $J_{i j}$ acyclic. Now we adjoin $R_{1}$ and $R_{2}$ at the upper endpoints and use the functor $\tilde{\varphi}$ of 2.4 to patch the zero complexes on $R_{1}$ and $R_{2}$ to the endpoints of $J_{i j}$. The maps among the $J_{i j}$ extend to the zero maps on the zero complexes. Call the new objects $H_{i j}$. 
We have now constructed objects $H_{i j}(0 \leqslant i \leqslant j)$ and structured triangles

$$
H_{i j} \rightarrow H_{i k} \rightarrow H_{j k}
$$

so that the $H_{i j}$ fit together to form an $n$-simplex in $\hat{T}_{.}^{\Phi}(R)$. Thus we have defined maps

$$
G_{n}^{\Phi}\left(R_{12}\right) \rightarrow \hat{T}_{n}^{\Phi}(R)
$$

for each $n$. It is very easy to check that these commute with all faces and degeneracies (after making the identifications called for in the description of $\hat{T}$ as a direct limit). Therefore, we have constructed the promised map of simplicial sets.

4.5. From the construction of 4.4 , we get a map

$$
K_{i}^{\Phi}\left(R_{12}\right) \stackrel{\partial}{\rightarrow} \hat{K}_{i-1}^{\Phi}(R)
$$

4.6. Mayer-Vietoris. Consider again the pullback square (4.1.1). Let $\Phi, \Phi_{1}, \Phi_{2}$ and $\Phi_{12}$ be families of supports satisfying the conditions in 4.1. For example, take an integer $m$, let $\Phi_{12}$ be the collection of all closed subsets in $\operatorname{Spec}\left(R_{12}\right)$ that have codimension $\geqslant m$, let $\Phi_{i}(i=1,2)$ be the collection of all closed sets in $\operatorname{Spec}\left(R_{i}\right)$ that have codimension $\geqslant m$ and restrict to elements of $\Phi_{12}$, and let $\Phi$ be the collection of all closed sets in $R$ that restrict to elements of $\Phi_{1}$ and $\Phi_{2}$.

We now specialize to the case where $R_{1}=R_{2}$, and since the subscripts are now confusing, denote this ring by $S$. Let $S^{(n)}$ be the pullback of the diagram

$$
S[t] \rightarrow S[t] \leftarrow S[t] \leftarrow \cdots \rightarrow S[t] \leftarrow S[t]
$$

in which $S[t]$ occurs $n$ times, the leftward arrows take $t$ to 0 , and the rightward arrows take $t$ to 1 . The ring $R^{(n)}$ of Section 4.1 is naturally a quotient of $S^{(n)}$; so there are natural maps $K_{0}^{\Phi}\left(S^{(n)}\right) \rightarrow \hat{K}_{0}^{\Phi}(R)$. We define $\hat{K}_{0}^{\Phi}(R)^{\prime}$ to be the quotient of $\hat{K}_{0}^{\Phi}(R)$ by the sum of the images of these maps.

There is then a sequence of maps

$$
K_{1}^{\Phi}\left(R_{12}\right) \stackrel{\partial}{\rightarrow} \hat{K}_{0}^{\Phi}(R) \rightarrow K_{0}^{\Phi}\left(R_{1}\right) \oplus K_{0}^{\Phi}\left(R_{2}\right) \rightarrow K_{0}^{\Phi}\left(R_{12}\right)
$$

with $\partial$ induced by 4.5 . (We now revert to writing $R_{1}$ and $R_{2}$ for the two copies of $S$ since it will be important to distinguish them.)

Let $K_{0}^{\Phi}(R)^{\prime}$ be the image of $K_{0}^{\Phi}(R)$ in $\hat{K}_{0}^{\Phi}(R)^{\prime}$ and consider the diagram

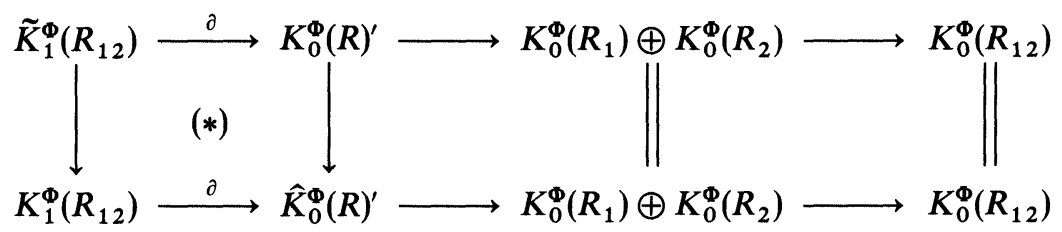


where $\tilde{K}_{1}^{\Phi}\left(R_{12}\right)$ and the map $\partial: \tilde{K}_{1}^{\Phi}\left(R_{12}\right) \rightarrow K_{0}^{\Phi}(R)^{\prime}$ are defined to make $(*)$ a pullback square. The top row of this diagram will be called the Mayer-Vietoris sequence associated to the square (4.1.1).

THEOREM. If $R_{1}=R_{2}$, then the Mayer-Vietoris sequence associated to (4.1.1) is exact.

Proof. We need to check exactness at $K_{0}^{\Phi}(R)^{\prime}$. An element of the kernel can be represented by a complex of projective objects $A$. on $R$. Let $A_{i}$ be the restriction of $A$. to $R_{i}$. Then each $\left[A_{i \cdot}\right]=0$ in $K_{0}^{\Phi}\left(R_{i}\right)$, so that if we use $\gamma$ to identify $R_{1}$ and $R_{2}$ we can say in particular that $\left[A_{1}.\right]=\left[A_{2}.\right]$.

Consequently, adding a common summand to the $A_{i}$ if necessary and invoking the trick of Heller [He], we may assume that there are triangles

$$
\begin{aligned}
& X_{.} \rightarrow A_{1} \cdot \rightarrow Y . \\
& X_{\bullet} \rightarrow A_{2 \cdot} \rightarrow Y .
\end{aligned}
$$

in $\mathscr{C}_{b}^{\Phi}\left(\mathbf{P}\left(R_{1}\right)\right)$. Restricting these triangles to $R_{12}$ gives triangles that we denote by

$$
\begin{aligned}
& \bar{X}_{\bullet} \rightarrow \overline{A_{1}} \cdot \rightarrow \bar{Y}_{\bullet} \\
& \bar{X}_{\bullet} \rightarrow \bar{A}_{2 \bullet} \rightarrow \bar{Y}_{\bullet} .
\end{aligned}
$$

The original complex $A_{0}$ determines an isomorphism $\overline{A_{1}} \cdot \stackrel{\alpha}{\Rightarrow} \overline{A_{2}}$.

The following three pairs of triangles describe one-simplices that form a loop in $G^{\Phi}\left(R_{12}\right)$ :

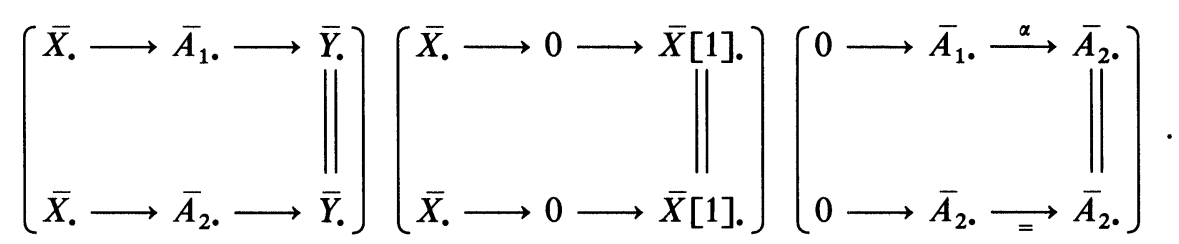

The image of this loop is easily seen to be $[A.] \in K_{0}^{\Phi}(R)^{\prime}$.

\section{REFERENCES}

[AC] S. LANDSBURG, Algebraic cycles relative to a smooth principal divisor, Comm. Alg. 16 (1988), 859-868.

[GG] H. Gillet AND D. Grayson, The loop space of the Q-construction, Ill. J. Math. 31 (1987), 574-597.

[He] A. HELLER, Some exact sequences in algebraic K-theory, Topology 3 (1965).

[HS] V. A. HiNich AND V. V. SCHECHTMAN, Geometry of a category of complexes and algebraic K-theory, Duke Math. J. 52 (1985), 399-430. 
[KTDC] S. LANDSBURG, $K$-theory and derived categories, unpublished.

[M] J. MiLnOR, Introduction to Algebraic K-Theory, Princeton Univ. Press, Princeton, 1971.

[Ma] S. H. MAN, Modules of finite homological dimension over a pullback of commutative noetherian rings, preprint.

[PM] S. LANDSBurg, Patching modules of finite projective dimension, Comm. Alg. 13 (1985), 1461-1473.

[RCAKT] - Relative cycles and algebraic K-theory, Amer. J. Math. 111 (1989), 599-632.

[RCG] , Relative Chow groups, preprint.

[T] R. Thomason And T. Trobaugh, Higher algebraic K-theory of schemes and of derived categories, preprint.

[W] F. WALDhAuSEN, “Algebraic K-Theory of spaces" in Algebraic and Geometric Topology, Springer Lecture Notes 1126 (1985), 318-419.

Department of Mathematics, Colorado State University, Fort Collins, Colorado 80523 